\title{
Commutativity of missing label operators in terms of Berezin brackets
}

\author{
Luis J. Boya $\uparrow$ \\ $\dagger$ Dpto. Física Teórica \\ Facultad de Ciencias \\ Universidad de Zaragoza \\ E-50009 Zaragoza, Spain \\ E-mail: luisjo@unizar.es \\ Rutwig Campoamor-Stursberg $\ddagger$ \\ $\ddagger$ Dpto. Geometría y Topología \\ Fac. CC. Matemáticas \\ Universidad Complutense de Madrid \\ Plaza de Ciencias, 3 \\ E-28040 Madrid, Spain \\ E-mail: rutwig@mat.ucm.es
}

\begin{abstract}
We obtain a criterion on the commutativity of polynomials in the enveloping algebra of a Lie algebra in terms of an involution condition with respect to the Berezin bracket. As an application, it is shown that the commutativity requirement of missing label operators for reduction chains in the missing label problem can be solved analytically.
\end{abstract}

PACS numbers: $02.20 \mathrm{~Sv}, 21.60 \mathrm{Fw}$ 


\section{Introduction}

A classical situation where group theory is applied to the description of a physical system is concerned with classification schemes. Here, irreducible representations of an (approximate) symmetry Lie group must be decomposed into irreducible representations of a certain subgroup in order to classify states:

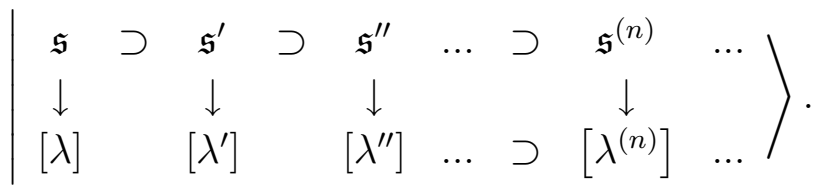

Depending on the situation, the labels obtained from the reduction (1) are sufficient to solve the problem if we require multiplicity free reductions, as happens for various models in nuclear physics [1]. However, for non-multiplicity free reductions, the subgroup does not always provide a sufficient number of labels to characterize the basis states without ambiguity. This happens in many of the non-canonical embeddings and generic irreducible representations (IRREPs) of Lie algebras. This is not necessarily a constraint, since often the interesting representations belong to a certain type, and degeneracies are solved directly with the available Casimir operators.

Many different approaches to solve the so-called missing label problem (short MLP) have been proposed in the literature, varying from projection of states to the obtainment of subgroup scalars in the enveloping algebras [2, 3, 4, 5, 6, 7]. Even if the latter procedure allows to find the most general labelling operator, the effective computation of integrity bases $\ddagger$ is a rather complicated problem, and no effective method is available. Among the difficulties appearing in this approach, we remark that no general criterion to decide how many operators are necessary to generate an integrity basis is known. Further, it should be expected that labelling operators have some interpretation in a physical context, as happens for the Elliott chain $\mathfrak{s u}(3) \supset \mathfrak{s o}(3)$ used in nuclear physics or the Racah chain $\mathfrak{s o}(7) \supset G_{2} \supset \mathfrak{s o}(3)$ used in the description of $f$-electron configurations [1, 8, 9]. Using the original conception of Lie groups as groups of transformations with their infinitesimal generators, an analytical approach using differential equations is possible, and easily adaptable to the MLP [10]. From this perspective, labelling operators can be seen as particular solutions of a certain subsystem of partial differential equations corresponding to an embedded subalgebra. Classical operators are recovered easily using the symmetrization map for tensors.

The labelling problem is, to some extent, related to symmetry breaking, and it seems therefore reasonable that contractions of Lie algebras play some role [11]. This ansatz was used in [12] to observe that any reduction chain $\mathfrak{s} \supset \mathfrak{s}^{\prime}$ is naturally related to some types of inhomogeneous Lie algebras obtained by a contraction procedure. This approach sufficed to generate physically interesting missing label, and allowed to solve intrinsically some MLP, like those with one labelling operator and others with a higher

$\ddagger$ An integrity basis is formed by a finite number of elementary labelling operators in terms of which all others may be written as products. 
number. Expansions of this procedure were developed in [13], where Casimir operators were decomposed with respect to the contraction related to the MLP. The problem of generating commuting missing label operators remained however open.

The main objective of this article is to enlarge the previous work of [12, 13], proving that commutativity of labelling operators can be solved using an analytical approach. To this extent, we have to consider a generalized Poisson bracket in the space

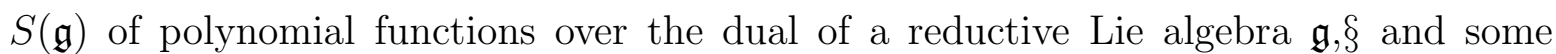
identities developed in [14] concerning the relation of generalized Poisson brackets and the commutator in the enveloping algebra $\mathcal{U}(\mathfrak{g})$ via the standard symmetrization map. In this sense, commuting labelling operators will correspond to unsymmetrized labelling operators that are in involution with respect to this generalized Poisson bracket.

As example to the procedure, we obtain three commuting labelling operators for the chain $\mathfrak{s p}(6) \supset \mathfrak{s u}(3) \times \mathfrak{u}(1)$ used in the symplectic nuclear collective model. We stress that, although an integrity basis for this MLP was derived in [15], no explicit solution to this MLP has been constructed.

\section{Missing label operators}

It is well known from classical theory that any semisimple Lie algebra $\mathfrak{g}$ possesses exactly $\mathcal{N}(\mathfrak{g})=l$ independent Casimir operators, i.e., polynomials in the generators that commute with all elements of the algebra, where $l$ denotes the rank of the algebra. The eigenvalues of Casimir operators are used to label without ambiguity the irreducible representations of $\mathfrak{g}$, while the states within a multiplet can be distinguished using the Cartan subalgebra. In some situations, however, these operators are not enough to separate multiplicities, and need additional operators to completely classify states. As shown in [16], the total number of internal labels required is thus

$$
i=\frac{1}{2}(\operatorname{dim} \mathfrak{g}-\mathcal{N}(\mathfrak{g})) .
$$

A similar situation holds whenever we use some subalgebra $\mathfrak{h}$ to label the basis states of irreducible representations of a Lie algebra $\mathfrak{g}$. The subgroup provides $\frac{1}{2}(\operatorname{dim} \mathfrak{h}+\mathcal{N}(\mathfrak{h}))+l^{\prime}$ labels, where $l^{\prime}$ is the number of invariants of $\mathfrak{g}$ that depend only on variables of the subalgebra $\mathfrak{h}[10$. To separate states within irreducible representations of $\mathfrak{g}$, we need to find

$$
n=\frac{1}{2}(\operatorname{dim} \mathfrak{g}-\mathcal{N}(\mathfrak{g})-\operatorname{dim} \mathfrak{h}-\mathcal{N}(\mathfrak{h}))+l^{\prime}
$$

additional operators, called missing label operators. The total number of available operators of this kind is easily shown to be twice the number of needed labels, i.e., $m=2 n$. For $n>1$, it remains the problem of determining a set of $n$ mutually commuting operators.

$\S$ We recall that a Lie algebra is called reductive if it is the direct sum of a semisimple and an Abelian Lie algebra. 
Although in general the missing label operators are neither invariants of the algebra nor any of its subalgebras, they can actually be determined by means of differential equations with the same ansatz as the general invariant problem [10, 17, 18, 19].

Given a Lie algebra $\mathfrak{g}=\left\{X_{1}, . ., X_{n} \mid\left[X_{i}, X_{j}\right]=C_{i j}^{k} X_{k}\right\}$ in terms of generators and commutation relations, classical Casimir operators are polynomials $C_{p}=\alpha^{i_{1} . i_{p}} X_{i_{1}} . . X_{i_{p}}$ in the generators of $\mathfrak{s}$ such that the constraint $\left[X_{i}, C_{p}\right]=0,(i=1 . . n)$ is satisfied. Therefore they are elements of the centre of the enveloping algebra $\mathcal{U}(\mathfrak{g})$ of $\mathfrak{g}$. In order to cover arbitrary Lie groups, it is convenient to use an analytical realization. The generators of the Lie algebra $\mathfrak{g}$ are realized in the space $C^{\infty}\left(\mathfrak{g}^{*}\right)$ by means of the differential operators:

$$
\widehat{X}_{i}=C_{i j}^{k} x_{k} \frac{\partial}{\partial x_{j}}
$$

where $\left\{x_{1}, . ., x_{n}\right\}$ are the components of a covector in a dual basis of $\left\{X_{1}, . ., X_{n}\right\}$. The invariants of $\mathfrak{g}$ (in particular, the Casimir operators) are then solutions of the following system of partial differential equations:

$$
\widehat{X}_{i} F=0, \quad 1 \leq i \leq n .
$$

For a polynomial solution of (5), the standard symmetrization map defined by

$$
\Lambda\left(x_{i_{1}} . . x_{i_{p}}\right)=\frac{1}{p !} \sum_{\sigma \in S_{p}} X_{\sigma\left(i_{1}\right)} . . X_{\sigma\left(i_{p}\right)}
$$

allows to recover the Casimir operators in their usual form, i.e, as elements in the centre of $\mathcal{U}(\mathfrak{g})$. The number $\mathcal{N}(\mathfrak{g})$ of functionally independent solutions of (5) is obtained from the classical criteria :

$$
\mathcal{N}(\mathfrak{g}):=\operatorname{dim} \mathfrak{g}-\operatorname{rank}\left(C_{i j}^{k} x_{k}\right),
$$

where $A(\mathfrak{g}):=\left(C_{i j}^{k} x_{k}\right)$ is the matrix associated to the commutator table of $\mathfrak{g}$ over the given basis.

If we now consider an algebra-subalgebra chain $\mathfrak{s} \supset \mathfrak{s}^{\prime}$ determined by an embedding $f: \mathfrak{s}^{\prime} \rightarrow \mathfrak{s}$, the missing label operators can be computed considering the equations of (5) corresponding to the generators of the subalgebra $\mathfrak{s}^{\prime}$. This system, as proven in [10], has exactly $\mathcal{N}\left(f\left(\mathfrak{s}^{\prime}\right)\right)=\operatorname{dim} \mathfrak{s}-\operatorname{dim} \mathfrak{s}^{\prime}-l^{\prime}$ solutions. Using formula (3) it follows further that $\mathcal{N}\left(f\left(\mathfrak{s}^{\prime}\right)\right)$ can be expressed in terms of the number of invariants of the algebra-subalgebra chain:

$$
\mathcal{N}\left(f\left(\mathfrak{s}^{\prime}\right)\right)=m+\mathcal{N}(\mathfrak{s})+\mathcal{N}\left(\mathfrak{s}^{\prime}\right)-l^{\prime}
$$

This shows that the differential equations corresponding to the subalgebra generators have exactly $n$ more solutions as needed to solve the missing label problem, as expected. Even using the analytical approach, to find a complete set of solutions for the labelling problem is a non-trivial task. 


\section{Commuting polynomials in enveloping algebras}

In this section we show a commutation property of polynomials in enveloping algebras that will help us later to find an analytical criterion to check whether two given labelling operators commute.

Let $\mathfrak{g}$ be a Lie algebra and $\mathcal{U}(\mathfrak{g})$ its enveloping algebra. Further let $S(\mathfrak{g})$ denote the space of polynomials defined on the dual space $\mathfrak{g}^{*}$. The standard symmetrization of monomials (6) can be easily extended to a one-to-one linear map

$$
\Lambda: S(\mathfrak{g}) \rightarrow \mathcal{U}(\mathfrak{g}) .
$$

In particular, if $x_{k_{1}} . x_{k_{p}}$ is a monomial of degree $p$, then for any $\sigma \in S_{p}$ we have the identity

$$
\Lambda\left(x_{k_{1}} \ldots x_{k_{p}}\right)=\Lambda\left(x_{\sigma\left(k_{1}\right)} . . x_{\sigma\left(k_{p}\right)}\right) .
$$

Conversely, given a polynomial $P=c^{k_{1} \ldots, k_{n}} X_{k_{1}} \ldots X_{k_{n}} \in \mathcal{U}(\mathfrak{g})$, we find its analytical counterpart $\pi(P)=P^{\prime}=c^{k_{1} . . k_{n}} x_{k_{1}} . x_{k_{n}}$ by simply replacing the generator $X_{i}$ by the corresponding variable $x_{i}$ of $\mathfrak{g}^{*}$.

It is well known that $\mathcal{U}(\mathfrak{g})$ is naturally filtered [20]. For any positive integer $n$, let $\mathcal{U}_{n}(\mathfrak{s})$ be the subspace of $\mathcal{U}(\mathfrak{g})$ generated by the products $X_{1} \ldots X_{p}$, where $p \leq n$. Clearly $\mathcal{U}_{n}(\mathfrak{g}) \subset \mathcal{U}_{n+1}(\mathfrak{g})$ for all $n$, and $\mathcal{U}(\mathfrak{g})=\bigcup_{n} \mathcal{U}_{n}(\mathfrak{g})$. Given an element $u \in \mathcal{U}(\mathfrak{g})$, we say that it has filtration $p$ if $u \in \mathcal{U}_{p}(\mathfrak{g})$ and $u \notin \mathcal{U}_{p-1}(\mathfrak{g})$.

It should be observed that, even if $\pi(u)=\pi(v)$, the elements $u$ and $v$ do not necessarily commute. Taking e.g., $u=X_{1} X_{2}$ and $v=X_{2} X_{1}$, a routine verification shows that

$$
\begin{gathered}
{[u, v]=-\left[X_{2}, X_{1}\right] X_{2} X_{1}-\left[X_{1}, X_{2}\right] X_{2} X_{1}-\left[X_{2},\left[X_{1}, X_{2}\right]\right] X_{1}-X_{2}\left[X_{1},\left[X_{2}, X_{1}\right]\right]} \\
=-\left[X_{2},\left[X_{1}, X_{2}\right]\right] X_{1}-X_{2}\left[X_{1},\left[X_{2}, X_{1}\right]\right] .
\end{gathered}
$$

This is an immediate consequence of the fact that the variables appearing are the same, but in reverse order. This situation can be generalized in straightforward manner to an arbitrary number of generators, covering also the case where $\pi(u) \neq \pi(v)$.

Lemma 1 Let $P=X_{1} . . X_{r} P_{1} \in U_{p}(\mathfrak{g}), Q=X_{r} . . X_{1} Q_{1} \in U_{q}(\mathfrak{g})$ such that $\left[P_{1}, Q_{1}\right]=0$ and $\left[X_{i}, P_{1}\right]=\left[X_{i}, Q_{1}\right]=0$ for any $r=1, . .$, . Then the commutator satisfies $[P, Q] \in U_{p+q-2}(\mathfrak{g})$ and $[P, Q] \notin U_{p+q-1}(\mathfrak{g})$.

As remarked, this special case is not very surprising, since products of this kind are degenerate in some sense. Actually, reordering the first $r$ generators of $Q$, we can rewrite this polynomial as $Q=X_{1} \ldots X_{r} Q_{1}-$ $\sum_{k, l=1 . . r} X_{r} \ldots \widehat{X}_{k} \widehat{X}_{l}\left[X_{l}, X_{k}\right] \ldots X_{1} Q_{1}$. This shows at once that the commutator reduces to $[P, Q]=-\sum_{k, l=1 . . r}\left[X_{1} . . X_{r}, X_{r} \ldots \widehat{X}_{k} \widehat{X}_{l}\left[X_{l}, X_{k}\right] \ldots X_{1}\right] P_{1} Q_{1}$, thus $[P, Q] \in U_{p+q-2}(\mathfrak{g})$. Observe in particular that, in this case, we have that the projections $\pi(P)$ and $\pi(Q)$ share a common factor. For the situation that interests us, however, such products are very unlikely to appear, thus in the following we exclude them of our analysis. We shall 
say that a pair of monomials $P=X_{i_{1}} \ldots X_{i_{p}} \in \mathcal{U}_{p}(\mathfrak{g}), Q=X_{j_{1}} \ldots X_{j_{q}} \in \mathcal{U}_{q}(\mathfrak{g})$ such that $\pi(P) \neq \pi(Q)$ is factorizable if they can be written in the form

$$
P=X_{1}^{a_{1}} . . X_{l}^{a_{l}} P_{1} \in U_{p}(\mathfrak{s}), Q=X_{l}^{a_{l}} . . X_{1}^{a_{1}} Q_{1} \in U_{q}(\mathfrak{s}),
$$

where $\left[P_{1}, Q_{1}\right]=0$ and $\left[X_{i}, P_{1}\right]=\left[X_{i}, Q_{1}\right]=0, i=1, . ., l$. The pair $P, Q$ is nonfactorizable if no decomposition of the preceding type exists.

Lemma 2 Let $P=X_{i_{1}} \ldots X_{i_{p}} \in \mathcal{U}_{p}(\mathfrak{g})$ and $Q=X_{j_{1}} \ldots X_{j_{q}} \in \mathcal{U}_{q}(\mathfrak{g})$ be non-factorizable pair. If $[P, Q] \neq 0$, then $[P, Q]$ has at least one term of filtration $p+q-1$.

Proof. By the properties of the filtration in enveloping algebras, we have that $P Q$ and $Q P$ have filtration $p+q$, and further that

$$
[P, Q]=X_{i_{1}} \ldots X_{i_{p}} X_{j_{1}} \ldots X_{j_{q}}-X_{j_{1}} \ldots X_{j_{q}} X_{i_{1}} \ldots X_{i_{p}} \in \mathcal{U}_{p+q-1}(\mathfrak{g}) .
$$

Expanding this product with the usual rule, we arrive at a sum of the type

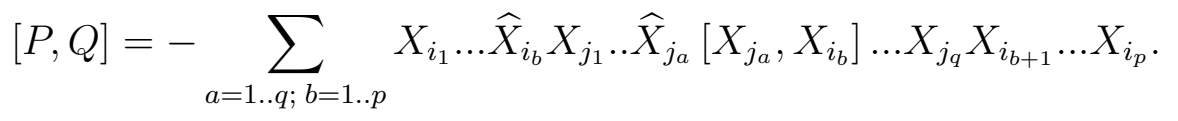

By assumption, after reordering the sum using the Poincaré-Birkhoff-Witt theorem, all terms of filtration $p+q-1$ must cancel. We argue basing on the number of different generators in the product $P Q=X_{i_{1}} \ldots X_{i_{p}} X_{j_{1}} \ldots X_{j_{q}}$ (which coincides with that of $Q P$ ). Suppose there are exactly $k \leq p+q$ different generators $X_{1}, . ., X_{k}$ in $P Q$. We further define $m_{t}$ as the multiplicity of the generator $X_{t}$ in the product $P Q$. Then any generator appearing in $Q P$ and $P Q$ equals some $X_{t}$ for $t=1, . ., k$. It is obvious that if $X_{j_{b}}=X_{i_{a}}$, then $\left[X_{j_{b}}, X_{i_{a}}\right]=0$ and the term $X_{i_{1}} \ldots \widehat{X}_{i_{b}} X_{j_{1}} . \widehat{X}_{j_{a}}\left[X_{j_{a}}, X_{i_{b}}\right] \ldots X_{j_{q}} X_{i_{b+1}} \ldots X_{i_{p}}$ vanishes. Thus in order to evaluate the commutator $[P, Q]$, we only have to consider the products $\left[X_{j_{a}}, X_{i_{b}}\right] \neq 0$. We now group together all terms involving generators with the same multiplicity, i.e., for any $X_{\alpha}, X_{\beta} \in\left\{X_{1}, . ., X_{k}\right\}$ with $X_{\alpha} \neq X_{\beta}$ we define

$$
\Phi_{[\alpha, \beta]}=\sum_{j_{a}, i_{b}} X_{i_{1}} \ldots \widehat{X}_{i_{b}} X_{j_{1}} . \widehat{X}_{j_{a}}\left[X_{j_{a}}, X_{i_{b}}\right] \ldots X_{j_{q}} X_{i_{b+1}} \ldots X_{i_{p}}
$$

where either $X_{j_{a}}=X_{\alpha}, X_{i_{b}}=X_{\beta}$ or $X_{j_{a}}=X_{\beta}, X_{i_{b}}=X_{\alpha}$. With the help of these polynomials, the commutator $[P, Q]$ can be expressed as $[P, Q]=-\sum_{1 \leq \alpha, \beta \leq k} \Phi_{[\alpha, \beta]}$. Reordering each term $\Phi_{[\alpha, \beta]}$ with respect to some basis of $\mathfrak{g}, \Phi_{[\alpha, \beta]}$ can be rewritten as

$$
\Phi_{[\alpha, \beta]}=\lambda_{(\alpha, \beta)} X_{i_{1}} \ldots \widehat{X}_{i_{b}} X_{j_{1}} . \widehat{X}_{j_{a}}\left[X_{j_{a}}, X_{i_{b}}\right] \ldots X_{j_{q}} X_{i_{b+1}} \ldots X_{i_{p}}+\text { L.O.T. }
$$

where $\lambda_{(\alpha, \beta)} \geq 0$ is an integer. The only possibility that $\lambda_{(\alpha, \beta)}=0$ occurs is that the commutator $\left[X_{j_{a}}, X_{i_{b}}\right]$ appears with the same multiplicity as $\left[X_{i_{b}}, X_{j_{a}}\right]$. This means that the generators $X_{j_{a}}, X_{i_{b}}$ appear in both $P$ and $Q$ with the same multiplicity, but in reverse order. By assumption, $\pi(P) \neq \pi(Q)$, thus there exists at least one generator $X_{l} \in\left\{X_{1}, . ., X_{k}\right\}$ such that its multiplicity in $P$ is different from that in $Q \llbracket$ This ensures the existence of one pair of indices $\alpha, \beta$ such that $\lambda_{(\alpha, \beta)}>0$. In this case, the

\| This also covers the case where a generator appears in $P$ and not in $Q$, or conversely. 
term of filtration $p+q-1$ does not vanish unless $\left[X_{j_{a}}, X_{i_{b}}\right]=0$. Discarding the zero terms, $[P, Q]$ is expressed as follows as a linear combination of basis elements:

$$
[P, Q]=-\sum_{1 \leq \alpha, \beta \leq k} \lambda_{(\alpha, \beta)} X_{i_{1}} \ldots \widehat{X}_{i_{b}} X_{j_{1}} . \widehat{X}_{j_{a}}\left[X_{j_{a}}, X_{i_{b}}\right] \ldots X_{j_{q}} X_{i_{b+1}} \ldots X_{i_{p}}+\text { L.O.T. }
$$

where $\lambda_{(\alpha, \beta)} \neq 0$. Observe now that if for any pair $\left\{X_{\alpha}, X_{\beta}\right\}$ such that $\lambda_{(\alpha, \beta)} \neq 0$ we have that $\left[X_{\alpha}, X_{\beta}\right]=0$, then $P$ and $Q$, after some reordering, would admit a decomposition of type (10), and therefore be a factorizable pair, which contradicts the initial assumption on their structure. Thus there exists at least a pair of indices $\alpha, \beta$ in $\{1, . ., k\}$ such that $\lambda_{(\alpha, \beta)} \neq 0$ and $\left[X_{\alpha}, X_{\beta}\right] \neq 0$.

If $[P, Q]$ is a sum of products of at most $p+q-2$ elements, then the basis elements $X_{i_{1}} \ldots \widehat{X}_{i_{b}} X_{j_{1}} . . \widehat{X}_{j_{a}}\left[X_{j_{a}}, X_{i_{b}}\right] \ldots X_{j_{q}} X_{i_{b+1}} \ldots X_{i_{p}}$ of (14) give rise to the dependence relation

$$
-\sum_{1 \leq \alpha, \beta \leq k} \lambda_{(\alpha, \beta)} X_{i_{1}} \ldots \widehat{X}_{i_{b}} X_{j_{1}} . . \widehat{X}_{j_{a}}\left[X_{j_{a}}, X_{i_{b}}\right] \ldots X_{j_{q}} X_{i_{b+1}} \ldots X_{i_{p}}=0
$$

We observe that each term of this sum has, leaving aside the commutator $\left[X_{j_{a}}, X_{i_{b}}\right]$, multiplicity $m_{t}$ in the generators $X_{t} \neq\left\{X_{\alpha}, X_{\beta}\right\}$, multiplicity $m_{\alpha}-1$ in $X_{\alpha}$ and $m_{\beta}-1$ in $X_{\beta}$, respectively. Let $\left[X_{j_{a}}, X_{i_{b}}\right]=C_{j_{a} i_{b}}^{t} X_{t} \in \mathfrak{g}$. If there exists some $t_{0}$ such that $X_{t_{0}} \notin\left\{X_{1}, . ., X_{k}\right\}$, then the term $\lambda_{(\alpha, \beta)} C_{j_{a} i_{b}}^{t_{0}} X_{i_{1}} \ldots \widehat{X}_{i_{b}} X_{j_{1}} . . \widehat{X}_{j_{a}} X_{t_{0}} \ldots X_{j_{q}} X_{i_{b+1}} \ldots X_{i_{p}}$ has multiplicity one in the generator $X_{t_{0}}$, and it follows at once by the multiplicity in the generators $\left\{X_{1}, . ., X_{k}\right\}$ that this term cannot be compensated with the remaining terms of (15). If $X_{t} \in\left\{X_{1}, . ., X_{k}\right\}$ for any $t$, then two possibilities are given:

(i) $X_{t_{0}}=X_{l}$ for some $l \neq \alpha, \beta$. Here $\lambda_{(\alpha, \beta)} C_{j_{a} i_{b}}^{t_{0}} X_{i_{1}} \ldots \widehat{X}_{i_{b}} X_{j_{1}} . \widehat{X}_{j_{a}} X_{t_{0}} \ldots X_{j_{q}} X_{i_{b+1}} \ldots X_{i_{p}}$ has multiplicity $m_{t}$ in the generators $X_{t} \neq\left\{X_{\alpha}, X_{\beta}, X_{l}\right\}$, multiplicity $m_{\alpha}-1$ in $X_{\alpha}, m_{\beta}-1$ in $X_{\beta}$ and $m_{l}+1$ in $X_{l}$, respectively. No other term in (15) has the same multiplicities, thus $[P, Q] \notin \mathcal{U}_{p+q-2}(\mathfrak{g})$.

(ii) $X_{t_{0}}=X_{j_{a}}$ ( or $X_{t_{0}}=X_{i_{b}}$ ). In this case, $\lambda_{(\alpha, \beta)} C_{j_{a} i_{b}}^{t_{0}} X_{i_{1}} \ldots \widehat{X}_{i_{b}} X_{j_{1}} . . \widehat{X}_{j_{a}} X_{t_{0}} \ldots X_{j_{q}} X_{i_{b+1}} \ldots X_{i_{p}}$ has multiplicity $m_{t}$ in the generators $X_{t} \neq\left\{X_{\beta}\right\}$ and multiplicity $m_{\beta}-1$ in $X_{\beta}$. Again, it is not possible that this term cancels with the remaining basis elements of (15), since their multiplicities in the generators are not the same.

Therefore, if the commutator does not vanish, there is at least one term of filtration $p+q-1$ that does not cancel.

\section{Berezin bracket}

In this section, using an an important relation between the commutator in the enveloping algebra $\mathcal{U}(\mathfrak{g})$ of a Lie algebra $\mathfrak{g}$ and a generalized Poisson bracket in $S(\mathfrak{g})$, we show that the commutativity requirement of labelling operators can be expressed, in the commutative frame, by means of an involution condition with respect to a special generalized Poisson bracket. This enables us to find an analytical characterization for missing label operators to commute mutually. 
Now let $f=c^{k_{1} . ., k_{p}} x_{k_{1}} . . x_{k_{p}} \in S(\mathfrak{g})$ be a given a homogeneous polynomial. It follows from (9) that writing its image $\Lambda(f)$ in symmetric form is unique. Moreover, choosing an arbitrary permutation $\sigma \in S_{p}$, we have that

$$
\begin{aligned}
\Lambda(f) & =\frac{1}{p !} \sum_{\nu \in \mathcal{S}_{p}} c^{k_{1 . . .}, k_{p}} X_{\nu\left(k_{1}\right)} . . X_{\nu\left(k_{p}\right)}=\left(c^{k_{1} . ., k_{p}} X_{k_{1}} . . X_{k_{p}}+\text { L.O.T. }\right) \\
& =\left(c^{k_{1} . ., k_{n}} X_{\sigma\left(k_{1}\right) . .} X_{\sigma\left(k_{p}\right)}+\text { L.O.T. }\right),
\end{aligned}
$$

where L.O.T. designates the terms of order $q \leq p-1$.

In [21] it was shown that for any $X_{i} \in \mathfrak{g}$ and any symmetrized polynomial $\Lambda(f)$, the commutator $\left[X_{i}, \Lambda(f)\right]$ in $\mathcal{U}(\mathfrak{g})$ corresponds, under the symmetrization map $\Lambda$, to a linear operator $A_{x_{i}}(f)$ in $S(\mathfrak{g})$, where $\Lambda\left(x_{i}\right)=X_{i}$. These operators enabled to solve the problem of finding a polynomial in $S(\mathfrak{g})$, the symmetrization $\Lambda(f)$ of which coincides with the commutator in $\mathcal{U}(\mathfrak{g})$ of two previously given symmetrized polynomials $g$ and $h$. More specifically, given two given (homogeneous) polynomials $g, h \in S(\mathfrak{g})$, the commutator $[\Lambda(g), \Lambda(h)]=\Lambda(g) \Lambda(h)-\Lambda(h) \Lambda(g)$ of their symmetrizations is the image, under $\Lambda$, of some polynomial $f \in S(\mathfrak{g}) \oplus$ A routine but long and tedious computation [21] shows that the latter polynomial $f \in S(\mathfrak{g})$ is given by the expression

$f=-C_{i j}^{k} x_{k} \frac{\partial g}{\partial x_{i}} \frac{\partial h}{\partial x_{j}}+F\left(x_{k}, \frac{\partial^{2} g}{\partial x_{i} \partial x_{j}}, \frac{\partial^{2} h}{\partial x_{j} \partial x_{i}}, \ldots, \frac{\partial^{p} g}{\partial x_{j_{1}} . . \partial x_{j_{p}}}, \frac{\partial^{p} h}{\partial x_{j_{1}} . . \partial x_{j_{p}}}, \ldots\right)$,

where $F$ is a polynomial the terms of which involve derivatives of order $d \geq 2$ of $g$ and $h[(\underline{20})$, equation (31)]. In particular, if $g, h$ are homogeneous of degrees $p$ and $q$, respectively, then $F$ can be decomposed as a sum of homogeneous polynomials of degrees $\leq p+q-2[21$.

Formula (17) already generalizes, in some sense, the analytical approach to compute Casimir operators of Lie algebras [10]. Observe that taking $g=x_{l}$, the preceding expression reduces to

$$
f=-C_{i j}^{k} x_{k} \frac{\partial x_{l}}{\partial x_{i}} \frac{\partial h}{\partial x_{j}}=-C_{l j}^{k} x_{k} \frac{\partial h}{\partial x_{j}}=\widehat{X}_{l}(h),
$$

where $F$ vanishes because of $\frac{\partial^{p} x_{l}}{\partial x_{j_{1}} . x_{j_{p}}}=0$ for any $p \geq 2$. In particular, if $h$ is a Casimir invariant of $\mathfrak{g}$, then $\widehat{X}_{l}(h)=0$, and by the symmetrization map $\Lambda$ we obtain that $\left[X_{l}, \Lambda(h)\right]=0+$ This fact suggests that (17) can be used to obtain an analytical criterion for the commutativity of labelling operators.

If we restrict to the important case where $g, h$ are homogeneous polynomials, then equation (17) can be rewritten as

$$
[\Lambda(g), \Lambda(h)]=\Lambda(\{g, h\})+\text { L.O.T. }
$$

9 Expressed in brackets, this condition is given by $[\Lambda(g), \Lambda(h)]=\Lambda(f)$.

+ This identification has already been used in the frame of completely integrable Hamiltonian systems [22. 
where

$$
\{g, h\}=-C_{i j}^{k} x_{k} \frac{\partial g}{\partial x_{i}} \frac{\partial h}{\partial x_{j}}
$$

is called the Berezin bracket of $g$ and $h$ and the lower order terms correspond to the symmetrization of the polynomial $F$ [14, 21]. It can further be shown that the Berezin bracket is a generalized Poisson bracket on the space $S(\mathfrak{g})[14,22]$. We observe that equation (19) is valid for more general Poisson brackets [14].

Let $F=c^{k_{1} \ldots k_{p}} X_{k_{1}} . . X_{k_{p}}, G=c^{j_{1} \ldots j_{q}} X_{j_{1}} . . X_{j_{q}}$ be two polynomials in the generators of $\mathfrak{g}$. Generalizing the situation of the previous section, we will say that $F, G$ forms a nonfactorizable pair if for any pair $\left\{c^{k_{1} . ., k_{p}}, c^{j_{1} . ., j_{q}}\right\}$ the monomials $\left\{X_{k_{1}} . . X_{k_{p}}, X_{j_{1}} . . X_{j_{q}}\right\}$ do not admit a decomposition of type (10).

Theorem 1 Let $F, G$ be a non-factorizable pair of polynomials in the enveloping algebra $\mathcal{U}(\mathfrak{g})$ of $\mathfrak{g}$ such that $F=\Lambda(f), G=\Lambda(g)$ for some homogeneous polynomials $f, g \in S(\mathfrak{g})$. Then $[F, G]=0$ if and only if $\{f, g\}=0$, i.e., if the functions $f, g$ are in involution with respect to the Berezin bracket.

Proof. Let $f=a^{k_{1} . ., k_{p}} x_{k_{1}} . . x_{k_{p}}$ and $g=b^{j_{1} ., j_{q}} x_{j_{1}} . . x_{j_{q}}$ be homogeneous polynomials of degrees $p$ and $q$, respectively, and let $\Lambda(f)=\frac{1}{p !} \sum_{\sigma \in \mathcal{S}_{p}} c^{k_{1} . ., k_{p}} X_{\sigma\left(k_{1}\right)} \ldots X_{\sigma\left(k_{p}\right)}$ and $\Lambda(g)=\frac{1}{q !} \sum_{\sigma \in \mathcal{S}_{q}} c^{j_{1} . ., j_{q}} X_{\sigma\left(j_{1}\right)} . . X_{\sigma\left(j_{q}\right)}$ be their symmetrizations. Suppose that their commutator in $\mathcal{U}(\mathfrak{g})$ vanishes:

$$
[\Lambda(f), \Lambda(g)]=0 .
$$

By homogeneity, formula (19) gives rise to the identity

$$
[\Lambda(f), \Lambda(g)]=\Lambda(\{f, g\})+\text { L.O.T. }=0
$$

Since the Berezin bracket $\{f, g\}=C_{i j}^{k} x_{k} \frac{\partial f}{\partial x_{i}} \frac{\partial g}{\partial x_{j}}$ is a homogeneous polynomial of degree $p+q-1$, the symmetric representative of $\Lambda(\{f, g\})$ is unique $*$ thus the vanishing of the commutator (21) implies in particular that $\Lambda(\{f, g\})=0$, because of the injectivity of (91), and therefore that

$$
\{f, g\}=0 .
$$

This proves that $f$ and $g$ are in involution with respect to the Berezin bracket. Conversely, let $f$ and $g$ be homogeneous polynomials such that the constraint

$$
\{f, g\}=C_{i j}^{k} x_{k} \frac{\partial f}{\partial x_{i}} \frac{\partial g}{\partial x_{j}}=0
$$

holds. It follows from the definition of the symmetrization map (9) that for any monomial $x_{1} \ldots x_{k}$

$$
\Lambda\left(x_{1} \ldots x_{k}\right)=X_{1} \ldots X_{k}+\text { L.O.T. }
$$

\footnotetext{
* It is at this step where the homogeneity is essential for the validity of the argument, since equation (19) does not necessarily hold if homogeneity is not given.
} 
This means that we can rewrite the symmetrized polynomials $\Lambda(f)$ and $\Lambda(g)$ as

$$
\begin{aligned}
& \Lambda(f)=\frac{1}{p !} \sum_{\sigma \in \mathcal{S}_{p}} a^{j_{1} ., j_{p}} X_{\sigma\left(j_{1}\right) . . X_{\sigma\left(j_{p}\right)}}=\left(a^{j_{1} \ldots, j_{p}} X_{j_{1}} . . X_{j_{p}}+\text { L.O.T. }\right), \\
& \Lambda(g)=\frac{1}{q !} \sum_{\sigma \in \mathcal{S}_{q}} b^{k_{1} . ., k_{q}} X_{\sigma\left(k_{1}\right)} . . X_{\sigma\left(k_{q}\right)}=\left(b^{k_{1} . ., k_{q}} X_{k_{1}} . . X_{k_{q}}+\text { L.O.T. }\right) .
\end{aligned}
$$

Evaluating the commutator in the enveloping algebra gives

$$
\begin{aligned}
{[\Lambda(f), \Lambda(g)] } & =\left[a^{j_{1} . ., j_{p}} X_{j_{1}} \ldots X_{j_{p}}+\text { L.O.T., } b^{k_{1} . ., k_{q}} X_{k_{1}} . X_{k_{q}}+\text { L.O.T. }\right] \\
& =\left(a^{j_{1} \ldots, j_{p}} b^{k_{1} \ldots, k_{q}}\left[X_{j_{1}} . . X_{j_{p}}, X_{k_{1}} . . X_{k_{q}}\right]+\text { L.O.T. }\right) .
\end{aligned}
$$

By formula (19), the commutator has no terms of degree $p+q-1$, and since $\left\{X_{j_{1}} . . X_{j_{p}}, X_{k_{1}} . . X_{k_{q}}\right\}$ is a non-factorizable pair, it follows from Lemma 2 that

$$
a^{j_{1} \ldots, j_{p}} b^{k_{1} \ldots, k_{q}}\left[X_{j_{1}} . X_{j_{p}}, X_{k_{1}} . X_{k_{q}}\right]=0 .
$$

Now observe that, since $\Lambda(f)$ and $\Lambda(g)$ are the symmetrization of homogeneous polynomials, we can arbitrarily choose permutations $\theta_{1} \in S_{p}$ and $\theta_{2} \in S_{q}$ such that

$\Lambda(f)=\frac{1}{p !} \sum_{\sigma \in \mathcal{S}_{p}} a^{j_{1} \ldots, j_{p}} X_{\sigma\left(j_{1}\right)} . . X_{\sigma\left(j_{p}\right)}=\left(a^{j_{1} \ldots, j_{p}} X_{\theta_{1}\left(j_{1}\right)} . . X_{\theta_{1}\left(j_{p}\right)}+\right.$ L.O.T. $)$,
$\Lambda(g)=\frac{1}{q !} \sum_{\sigma \in \mathcal{S}_{q}} b^{k_{1} \ldots, k_{q}} X_{\sigma\left(k_{1}\right)} . . X_{\sigma\left(k_{q}\right)}=\left(b^{k_{1} . ., k_{q}} X_{\theta_{2}\left(k_{1}\right)} . . X_{\theta_{2}\left(k_{q}\right)}+\right.$ L.O.T. $)$.

Expanding the commutator $[\Lambda(f), \Lambda(g)]$ using these representatives, a reasoning identical to that above leads to the identity

$$
a^{j_{1} ., j_{p}} b^{k_{1} . ., k_{q}}\left[X_{\theta_{1}\left(j_{1}\right)} . . X_{\theta_{1}\left(j_{p}\right)}, X_{\theta_{2}\left(k_{1}\right)} . . X_{\theta_{2}\left(k_{q}\right)}\right]=0 .
$$

These identities enable us to sum over all permutations of $S_{p}$ and $S_{q}$, which leads to the equation

$$
\sum_{\sigma \in S_{p}} \sum_{\tau \in S_{q}} a^{j_{1} \ldots j_{p}} b^{k_{1} . ., k_{q}}\left[X_{\sigma\left(j_{1}\right)} . . X_{\sigma\left(j_{p}\right)}, X_{\tau\left(k_{1}\right)} . . X_{\tau\left(k_{q}\right)}\right]=0 .
$$

But observe that equation (31) is exactly the commutator of $\Lambda(f)$ and $\Lambda(g)$ if we use their symmetric representatives, from which we conclude that

$$
[\Lambda(f), \Lambda(g)]=0,
$$

showing that the labelling operators $F=\Lambda(f), G=\Lambda(g)$ commute.

We observe that, in the frame of Berezin brackets, equation (18) inherits meaning as a special case corresponding to linear polynomials. Therefore the analytical approach to compute Casimir operators can be seen as a particular application of the preceding result.

The main interest of the previous theorem relies in its application to the MLP, in order to check that two or more labelling operators commute with each other. Let $\mathfrak{s} \supset \mathfrak{s}^{\prime}$ be an algebra-subalgebra chain, where both $\mathfrak{s}$ and $\mathfrak{s}^{\prime}$ are reductive Lie algebras. 
Corollary 1 Let $F=\Lambda(f), G=\Lambda(g) \in \mathcal{U}(\mathfrak{s})$ be two non-factorizable missing label operators. Then $[F, G]=0$ if and only if $\{f, g\}=0$.

The requirement of reductive Lie algebras is imposed to guarantee the existence of a complete basis of labelling operators formed by polynomials [10]. In order to complete the analytical characterization, it should be justified that labelling operators are always non-factorizable, in order to prevent the degenerate case studied in the previous section. To this extent, suppose that $P, Q$ is a pair of labelling operators such that $[P, Q] \neq 0$ and $\{\pi(P), \pi(Q)\}=0$. By Lemmas 1 and $2, P$ and $Q$ would have the following shape:

$$
\begin{aligned}
& P=X_{\alpha_{1}^{1}} \ldots X_{\alpha_{p}^{1}} P_{1}+X_{\alpha_{1}^{2}} . . X_{\alpha_{q}^{2}} P_{2}+\ldots X_{\alpha_{1}^{r}} . . X_{\alpha_{s}^{r}} P_{r}+P^{\prime}, \\
& Q=X_{\alpha_{p}^{1}}, \ldots X_{\alpha_{1}^{1}} Q_{1}+X_{\alpha_{q}^{2}} . . X_{\alpha_{1}^{2}} Q_{2}+\ldots X_{\alpha_{s}^{r}} . . X_{\alpha_{1}^{r}} Q_{r}+Q^{\prime},
\end{aligned}
$$

where $\left[P^{\prime}, Q^{\prime}\right]=\left[P^{\prime}, Q_{i}\right]=\left[P_{i}, Q^{\prime}\right]=\left[P_{i}, Q_{i}\right]=0$ for $i=1, . ., r$ and $\left[X_{\alpha_{t}^{l}}, P_{k}\right]=$ $\left[X_{\alpha_{t}^{l}}, Q_{k}\right]=0$ for $l=1, . ., r ; k=1, . ., r$. As a consequence, the corresponding analytical counterparts $\pi(P), \pi(Q) \in \mathcal{S}(\mathfrak{g})$ would satisfy the relations

$$
\begin{aligned}
& \left\{\pi\left(P^{\prime}\right), \pi\left(Q^{\prime}\right)\right\}=\left\{\pi\left(P_{i}\right), \pi\left(Q_{i}\right)\right\}=\left\{\pi\left(P^{\prime}\right), \pi\left(Q_{i}\right)\right\}=\left\{\pi\left(P_{i}\right), \pi\left(Q^{\prime}\right)\right\}=0, \\
& \left\{x_{\alpha_{t}^{l}}, \pi\left(P_{k}\right)\right\}=\left\{x_{\alpha_{t}^{l}}, \pi\left(Q_{k}\right)\right\}=0,
\end{aligned}
$$

for $i=1, . . r ; l=1, . ., r ; k=1, . ., r$. On the other hand, both $\pi(P)$ and $\pi(Q)$ are functionally independent solutions to the subsystem of differential equations (5) corresponding to the generators of $\mathfrak{s}^{\prime}$, thus subjected to additional constraints. In practice, such degenerate independent labelling operators have never been observed $[2,3,4,5,7,18,12,13,15]$, and the large amount of conditions (33) that such operators should satisfy makes it unlikely that they are functionally independent. In any case, since the constraints (34) are not required by the reduction chain, we can always find independent labelling operators that are non-factorizable.

\subsection{Example: The chain $\mathfrak{s p}(6) \supset \mathfrak{s u}(3) \times \mathfrak{u}(1)$}

The unitary reduction of the non-compact symplectic Lie algebra $\mathfrak{s p}(6, \mathbb{R})$ has found applications in the nuclear collective model [23], where nuclear states are classified as bases of irreducible representations of $\mathfrak{s p}(6)$ reduced with respect to $\mathfrak{s u}(3) \times \mathfrak{u}(1)$. In this case, we have to add $n=3$ labelling operators to distinguish the states. Generating functions for this chain were studied in [15], which in particular allowed to derive an integrity basis consisting of 31 elementary subgroup scalars. However, the problem of extracting three commuting operators was not undertaken. We show that combining the decomposition of Casimir operators of [13] with the previous results, such a set of labelling operators can be obtained naturally.

To this extent, we use the Racah realization for the symplectic Lie algebra $\mathfrak{s p}(6, \mathbb{R})$ [16]. We consider the generators $X_{i, j}$ with $-3 \leq i, j \leq 3$ satisfying the condition

$$
X_{i, j}+\varepsilon_{i} \varepsilon_{j} X_{-j,-i}=0
$$


where $\varepsilon_{i}=\operatorname{sgn}(i)$. Over this basis, the brackets are given by

$$
\left[X_{i, j}, X_{k, l}\right]=\delta_{j k} X_{i l}-\delta_{i l} X_{k j}+\varepsilon_{i} \varepsilon_{j} \delta_{j,-l} X_{k,-i}-\varepsilon_{i} \varepsilon_{j} \delta_{i,-k} X_{-j, l}
$$

where $-3 \leq i, j, k, l \leq 3$. Using the polynomial block matrix $M$ defined by

$$
M=\left(\begin{array}{cc}
x_{i, j} & \sqrt{-1} x_{-i, j} \\
\sqrt{-1} x_{i,-j} & -x_{i, j}
\end{array}\right)
$$

and computing the coefficients of the characteristic polynomial [24]:

$$
\left|M-T \operatorname{Id}_{6}\right|=T^{6}+C_{2} T^{4}+C_{4} T^{2}+C_{6},
$$

we obtain three independent invariants $C_{2}, C_{4}$ and $C_{6}$ of $\mathfrak{s p}(6, \mathbb{R})$, and the symmetrized operators $\Lambda\left(C_{i}\right)$ give the usual Casimir operators in the enveloping algebra. As the unitary algebra $\mathfrak{u}(3)$ is generated by $\left\{X_{i, j} \mid 1 \leq i, j \leq 3\right\}$, it suffices to replace the diagonal operators $X_{i, i}$ by suitable linear combinations to obtain a $\mathfrak{s u}(3) \times \mathfrak{u}(1)$ basis. Taking $H_{1}=X_{1,1}-X_{2,2}, H_{2}=X_{2,2}-X_{3,3}$ and $H_{3}=X_{1,1}+X_{2,2}+X_{3,3}$ we get the Cartan subalgebra of $\mathfrak{s u}(3)$, while $H_{3}$ commutes with all $X_{i, j}$ with positive indices $i, j$. The invariants over the $\mathfrak{s u}(3) \times \mathfrak{u}(1)$ basis are obtained replacing the variables $x_{i, i}$ by the corresponding linear combinations of $h_{i}$ [24]. Now we construct labelling operators for this chain using the contraction method developed in [12]. The transformations determined by

$$
H_{i}^{\prime}=H_{i}, X_{i, j}^{\prime}=X_{i, j}, X_{-i, j}^{\prime}=\varepsilon X_{-i, j}, X_{i,-j}^{\prime}=\varepsilon X_{i,-j}
$$

define a contraction of $\mathfrak{s p}(6)$ onto the inhomogeneous algebra $(\mathfrak{s u}(3) \times \mathfrak{u}(1)) \vec{\oplus}_{R} 12 L_{1}$, where the representation $R$ decomposes into a sextet and antisextet with $\mathfrak{u}(1)$ weight \pm 1 and a singlet with $\mathfrak{u}(1)$ weight 1 . As shown in [13], this contraction induces a decomposition of the Casimir operators of $\mathfrak{s p}(6)$ The rescaled Casimir operators can be written as:

$$
\begin{aligned}
& C_{4}=\varepsilon^{4} C_{(4,0)}+\varepsilon^{2} C_{(2,2)}+C_{(0,4)}, \\
& C_{6}=\varepsilon^{6} C_{(6,0)}+\varepsilon^{4} C_{(4,2)}+\varepsilon^{2} C_{(2,4)}+C_{(0,6)},
\end{aligned}
$$

where $C_{(k, l)}$ denotes a homogeneous polynomial of degree $k$ in the variables of $R$ and degree $l$ in the variables of the unitary subalgebra. It can be shown that the $C_{(i, j)}$ are labelling operators [13], and that the $C_{(0, k)}$, being functions of the Casimir operators of $\mathfrak{s u}(3) \times \mathfrak{u}(1)$, do not provide independent labelling operators. The symmetrized operators of $C_{(2,2)}, C_{(4,2)}$ and $C_{(2,4)}$ are added to the Casimir operators of $\mathfrak{s p}(6)$ and the subalgebra $\mathfrak{s u}(3) \times \mathfrak{u}(1)$, and the resulting nine operators can be easily seen to be functionally independent. It is straightforward but time consuming to verify that these operators are non-factorizable. To solve the MLP, it remains to check the commutativity of the labelling operators. In order to determine whether the symmetrization of $C_{(2,2)}, C_{(4,2)}$ and $C_{(2,4)}$ commute, we compute the Berezin bracket. A routine but tedious computation shows that

$$
\left\{C_{(2,2)}, C_{(4,2)}\right\}=0, \quad\left\{C_{(2,2)}, C_{(2,4)}\right\}=0, \quad\left\{C_{(2,4)}, C_{(4,2)}\right\}=0 .
$$

$\sharp$ The quadratic operator is skipped since it provides no independent labelling operators [12, 13]. 
By Theorem 1, we conclude that

$$
\left[\Lambda\left(C_{(2,2)}\right), \Lambda\left(C_{(4,2)}\right)\right]=0, \quad\left[\Lambda\left(C_{(2,2)}\right), \Lambda\left(C_{(2,4)}\right)\right]=0, \quad\left[\Lambda\left(C_{(2,4)}\right), \Lambda\left(C_{(4,2)}\right)\right]=0 .
$$

We remark that a direct evaluation of the commutators of these symmetrized operators is a quite demanding computational problem, since the polynomials $C_{(2,2)}, C_{(2,4)}$ and $C_{(4,2)}$ have 126, 686 and 444 terms, respectively.

\section{Summary and outlook}

We have shown that the commutativity of labelling operators in the missing label problem can be solved using the analytical approach, by means of the so-called Berezin bracket, up to a special type of polynomials that is unlikely to appear in applications. We stress that none of the known labelling problems does admit solutions of such special type, and we firmly believe that do not appear as labelling operators, at least for reductive Lie algebras. However, even if such degenerate operators were possible, we can always find independent labelling operators that are non-factorizable, to which the analytical criterion is then applied. This result constitutes a natural enlargement of the classical analytical approach [10], and provides a general criterion to check the commutativity of labelling operators without being forced to determine first an integrity basis. Although the verification that two operators are non-factorizable is formally very simple, it can take a large amount of time depending on the number of terms.

The possibility of testing the commutativity of labelling operators in the analytical frame also opens new possibilities for the systematic search of the most general solution to a MLP. Potential applications of this procedure concern reduction chains for exceptional groups, as well as other high rank groups used in high energy physics [25], where an approach by means of enveloping algebras presents many computational problems. An analysis of these labelling problems systematized along these lines is currently in progress.

\section{Acknowledgment}

The authors express their gratitude to J C Moreno and the referee for useful discussions and valuable suggestions. During the preparation of this work, the authors were financially supported by the research projects FPA2006-02315 of the CICYT (LJB), MTM2006-09152 of the M.E.C. and CCG07-UCM/ESP-2922 of the U.C.M.-C.A.M. (RCS).

\section{References}

[1] Iachello F and Arima A 1987 The interacting boson model, (Cambridge Univ. Press, Cambridge).

[2] Elliott J P 1958 Proc. Roy. Soc. Lond. A 245 128, 562

Helmers K 1961 Nuclear Phys. 23594

Moshinsky M and Nagel J G 1963 Phys. Letters 5173 
Hecht K T 1994 J. Phys. A: Math. Gen. 273445

Jarvis P D 1974 J. Phys. A: Math. Gen. 71807

[3] Sharp R T and Pieper S C 1968 J. Math. Phys. 9663

Sharp R T 1975 J. Math. Phys. 16, 2050

[4] Judd B R, Miller W, Patera J and Winternitz P 1974 J. Math. Phys. 151787

Louck J D 1976 Proceedings of the International Symposium on Mathematical Physics, Mexico City, Mexico

Jarvis P D and Zhang R B 2005 J. Phys. A: Math. Gen. 38 L219

[5] Quesne Ch 1976 J. Math. Phys. 17, 1452; 18, 1210 (1977)

Partensky A and Maguin C 1978 J. Math. Phys. 19511

[6] Bincer A M 1983 J. Math. Phys. 241695

[7] Van der Jeugt 1984 J. Math. Phys. 251221

Hughes J W B and Van der Jeugt J 1985 J. Math. Phys. 26894

De Meyer H, Vanden Berghe G and De Wilde P 1987 Comput. Phys. Comm. 44197

[8] von Isacker P and Juillet O 1999 Nuclear Phys. A564 739

Valencia J P and Wu H C 2004 Braz. J. Phys. 34837

[9] Wybourne B G 1992 J. Phys. B: At. Mol. Opt. 251683

[10] Peccia A and Sharp R T 1976 J. Math. Phys. 171313

[11] Vitiello G and de Concini C 1976 Nuclear Phys. B116 141

[12] Campoamor-Stursberg 2007 J. Phys. A: Math. Theor. 4014773 (hep-th/arXiv:0706.2581)

[13] Campoamor-Stursberg R 2008 J. Phys. Conf. Ser. 128012052

[14] Ol'shanskii' G 1997 Transf. Groups 2197

[15] Gaskell R, Rosensteel G and Sharp R T 1981 J. Math. Phys. 222732

[16] Racah G 1949 Phys. Rev. $761352 — 1951$ Group Theory and Spectroscopy, (Princeton Univ. Press, New Jersey)

[17] Campoamor-Stursberg R 2007 J. Phys. A: Math. Theor. 405355

[18] Beltrametti E G and Blasi A 1966 Physics Letters 2062

[19] Abellanas L and Martínez Alonso L 1975 J. Math. Phys. 161580

[20] Dixmier J 1974 Algèbres enveloppantes (Gauthier-Villars, Paris)

[21] Berezin F A 1965 Metod vtorichnogo kvantovaniya (Nauka, Moscow)

-1967 Funkt. Anal. Prolozh. 11

[22] Perelomov A M 1990 Integriruemye sistemy klassicheskoi' mekhaniki i algebry Li (Glav. red. fizikomatematicheskoi' Lit-ry, Moscow)

[23] Graber J L and Rosensteel G 2003 Phys. Rev. C 68014301

[24] Campoamor-Stursberg R 2005 J. Phys. A: Math. Gen. 384187

[25] Wybourne B G 1995 Lithuanian J. Phys. 35123 